\title{
Viscosity of $\mathrm{Hg}_{0.84} \mathbf{Z n}_{\mathbf{0 . 1 6}}$ Te pseudobinary melt
}

\author{
K. Mazuruk, ${ }^{\text {a) }}$ Ching-Hua Su, Yi-Gao Sha, ${ }^{\text {a) }}$ and S. L. Lehoczky \\ Space Science Laboratory, NASA Marshall Space Flight Center, Alabama 35812
}

(Received 30 November 1995; accepted for publication 8 March 1996)

\begin{abstract}
An oscillating-cup viscometer was developed to measure viscosity of molten $\mathrm{HgZnTe}$ ternary semiconductor alloys. Data were collected for the pseudobinary $\mathrm{Hg}_{0.84} \mathrm{Zn}_{0.16} \mathrm{Te}$ melt between 770 and $850^{\circ} \mathrm{C}$. The kinematic viscosity was found to vary from approximately 1.1 to $1.4 \times 10^{-3}$ $\mathrm{cm}^{2} \mathrm{~s}^{-1}$. A slow relaxation phenomena was also observed for temperatures from the melting point of 770 to $\sim 800^{\circ} \mathrm{C}$. Possible mechanisms for this effect are discussed. (C) 1996 American Institute of Physics. [S0021-8979(96)03812-1]
\end{abstract}

\section{INTRODUCTION}

Experimental viscosity determinations of molten mercury-based semiconductor alloys are important for the development of crystal growth fundamentals. These melts are highly corrosive and have a high, basically mercury, vapor pressure which reaches approximately $60 \mathrm{~atm}$ at $900^{\circ} \mathrm{C}$. Therefore, handling and processing of such material becomes increasingly difficult. In fact, viscosities of $\mathrm{HgTe}$ and HgCdTe have been reported only recently. ${ }^{1}$

In this work, the viscosity of a $\mathrm{Hg}_{1-x} \mathrm{Zn}_{x} \mathrm{Te}$ melt was measured for the $\mathrm{ZnTe}$ molar fraction $x=0.16$ in the temperature range of $770-850^{\circ} \mathrm{C}$. An oscillating-cup method was used for this purpose with sealed thick-wall cylindrical silica ampoules employed as crucibles. While reproducibility of the data for temperatures above approximately $820^{\circ} \mathrm{C}$ is quite good when equilibration times of several hours were employed, measurements near the liquidus point of $770{ }^{\circ} \mathrm{C}$ show a very slow relaxation phenomenon which can last a week or even longer. The physical mechanism for this observed drift is not yet known but may be attributed to macro/ microscopic structural processes in the liquid phase.

A description of the experimental apparatus and the measurement technique is presented in Sec. II. The numerical analysis of the data and a critical discussion of the results comprises Sec. III.

\section{EXPERIMENT}

\section{A. Oscillating-cup viscometer}

The oscillation-cup technique ${ }^{2}$ is a highly suitable and commonly used technique for viscosity measurements of high temperature metallic melts. It has been recently implemented for the measurements of $\mathrm{HgTe}$ and $\mathrm{HgCdTe}$ melts ${ }^{\prime}$ and was utilized for the present study. A schematic view of the apparatus is depicted in Fig. 1 (for a detailed description see Ref. 1). Briefly, it consists of an oscillation unit suspended by a thin wire which provides the restoring torque. The suspension unit comprises a flywheel rigidly connected by the silica rod to the ampoule which is filled with the fluid under investigation; torsial oscillations take place about the vertical axis. Viscosity of the fluid causes the energy of the motion to dissipate slowly. Subsequently, logarithmic decre-

\footnotetext{
a)Also at: Universities Space Research Association.
}

ment of the oscillations is a measure of viscous dissipation and can be used to determine the absolute viscosity. The torque sensor, which consists of a strain gauge bridge assembly attached to the top end of the spring wire, was successfully utilized to measure the amplitude of the angular deflection of the oscillation motion. The signal from the strain gauges is recorded by a data acquisition system and stored for later processing. The suspended unit is housed within a thick-walled inconel tube for safety reasons, i.e., to contain occasional ampoule failures. The data acquisition rate was 10 readings per second, adequate for an oscillation period in the range of 5-10 s. The sensitivity of this type of sensor is a fraction of an angular degree. The temperature control of the three zone furnace is maintaining within $\pm 0.1^{\circ} \mathrm{C}$ and uniformity along the ampoule is approximately $\pm 2{ }^{\circ} \mathrm{C}$. The recorded data were subjected to numerical noise filtering before the actual determination of the logarithmic decrement. All the data recorded were accurately fitted to the exponential decay.

\section{B. Determination of viscosity}

The angular deflection of the oscillation-cup motion can be expressed as

$$
\alpha(t)=\alpha_{0} \exp \left(-\frac{\delta}{T} t\right) \cos \left(\omega t+\varphi_{0}\right),
$$

where $\omega=2 \pi / T$ is the angular frequency of the oscillation, $T$ its period, $t$ is time, $\alpha_{0}$ is the initial angular deflection, and $\delta$ is the logarithmic decrement of the motion. A logarithmic decrement $\delta_{0}$ of the suspension with an empty cup is essentially due to the internal friction of the wire. This term was measured and taken into account in the viscosity evaluation. In addition to the logarithmic decrements and frequency, the moment of inertia, I, of the suspension is required. This parameter was determined from the spring constant $G$. An inertia ring with an accurately known moment of inertia was used for this purpose; two angular frequencies of the oscillation with and without the ring were then measured. The spring constant and the moment of inertia can be obtained from the relation $G=I \omega^{2}$. Finally, mass $(M)$ and density $(\rho)$ of the fluid to measure, and the inner radius $(R)$ of the ampoule is also required. The viscosity can then be numerically determined from the exact theory $y^{3}$ or by employing consid- 


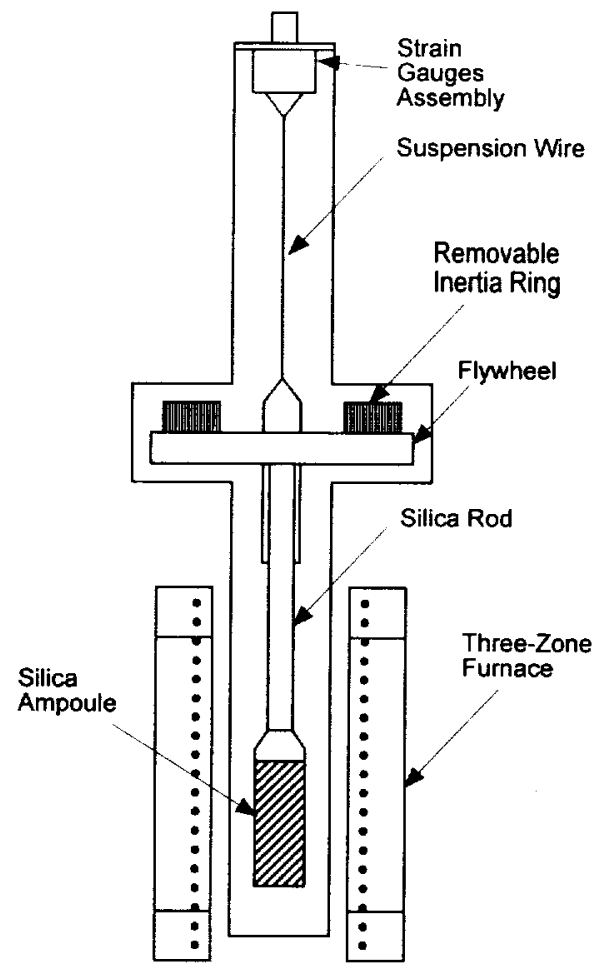

FIG. 1. Schematic view of the oscillating-cup viscometer.

erably simpler approximate equations. In our analysis we utilized the approximate Roscoe formula ${ }^{4}$ which can be written in the form

$$
\begin{aligned}
\Delta= & \frac{I^{\prime}}{I}\left[\left(1+\frac{R}{4 H}\right) \frac{a_{0} \sqrt{2}}{\xi_{0}}-\left(\frac{3}{2}+\frac{4 R}{\pi H}\right) \frac{2}{\xi_{0}^{2}}\right. \\
& \left.+\left(\frac{3}{8}+\frac{9 R}{4 H}\right) \frac{a_{2} \sqrt{2}}{\xi_{0}^{3}}\right],
\end{aligned}
$$

where,

$$
\begin{aligned}
& a_{0}=1-\frac{1}{2} \Delta-\frac{3}{2} \Delta^{2}, \quad a_{2}=1+\frac{1}{2} \Delta+\frac{1}{8} \Delta^{2}, \\
& I^{\prime}=M R^{2} / 2, \quad \xi_{0}=\sqrt{\omega / \nu} R, \quad \Delta=\frac{\delta-\delta_{0}}{2 \pi}, \\
& H=\frac{M}{\rho \pi R^{2}},
\end{aligned}
$$

and $\nu$ is the kinematic viscosity.

We have tested the accuracy of this formula against the exact theory and found very good agreement for the set of experimental parameters employed here. Figure 2 compares the curves obtained from the exact equations and the Roscoe formula. These curves can be used to determine $\xi_{0}$ from the experimentally obtained values of $\Delta$. In our experiments, the values of $\xi_{0}$ fall into the range of 18-25, where excellent agreement between the exact and the approximate approach is found ( $0.5 \%$ difference).

\section{Sample preparation and measurements}

Fused silica ampoules were cleaned and outgassed at $1000^{\circ} \mathrm{C}$ for $24 \mathrm{~h}$ under vacuum. Six-nine grade $\mathrm{Zn}$ and $\mathrm{Te}$

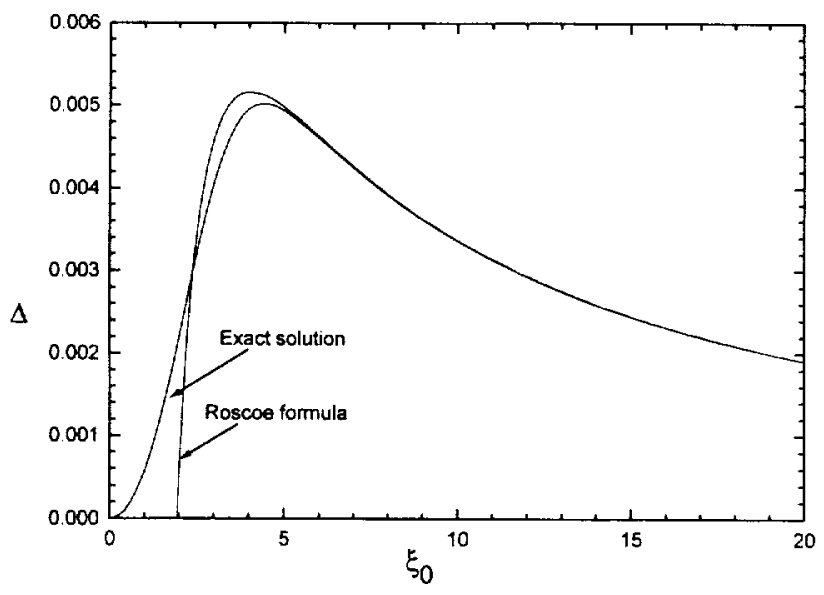

FIG. 2. Dependence of $\Delta_{0}$ on $\xi_{0}$ using the exact theory and the Roscoe formula. The input data set corresponds to sample II.

and seven-nine grade $\mathrm{Hg}$ were provided by Johnson Matthey. $\mathrm{Hg}_{1-x} \mathrm{Zn}_{x} \mathrm{Te}$ materials with $\mathrm{ZnTe}$ molar fraction of $x=0.16$ were synthesized in a rocking furnace according to the procedure developed in this laboratory. ${ }^{5}$ The first sample was prepared by a two-step procedure. After the sample was synthesized the ampoule was opened and a total of $118.64 \mathrm{gms}$ of $\mathrm{HgZnTe}$ was weighed and loaded into the viscosity ampoule which was then sealed under vacuum. As for the second sample, material synthesis and viscosity measurements were done in the same ampoule. The following amounts of material were loaded into the second ampoule: $45.100 \mathrm{~g}$ of $\mathrm{Hg}, 34.076 \mathrm{~g}$ of $\mathrm{Te}$ and $2.793 \mathrm{~g}$ of $\mathrm{Zn}$. The experimental parameters and properties for these two samples are given in Table I. Two different ampoule dimensions as well as two different operational parameters were employed to test consistency of the measurements.

Viscosity measurements were initiated by first determining the moment of inertia of the suspended system as well as the logarithmic decrement of the oscillation with solid material inside the ampoule. The sample temperature was gradually increased to $850^{\circ} \mathrm{C}$, the safety limit for the vapor pressure, and held for one day. Viscosity measurements were then taken at decreasing $5{ }^{\circ} \mathrm{C}$ temperature intervals, to the liquids point. The results of early experiments revealed an unexpected time drift of the logarithmic decrement at temperatures near the liquidus point. Consequently, the second

TABLE I. Experimental parameters and properties used in viscosity evaluation.

\begin{tabular}{lccc}
\hline \hline \multicolumn{1}{c}{ Parameter } & Sample I & Sample II & Units \\
\hline Spring constant, $G$ & 1034.63 & 1087.8 & $\mathrm{~g} \mathrm{~cm}^{2} / \mathrm{s}^{2}$ \\
Angular frequency, with & & & \\
$\quad$ empty ampoule, $\omega_{0}$ & 1.097 & 1.512 & $\mathrm{~s}^{-1}$ \\
Inner radius of ampoule, $R$ & 0.649 & 0.475 & $\mathrm{~cm}$ \\
Logarithmic decrement with & & & \\
$\quad$ empty ampoule, $\delta$ & 0.00315 & 0.00374 & \\
Sample mass, $M$ & 118.64 & 81.969 & $\mathrm{~g}$ \\
Melt density, $\rho$ & 7.476 & 7.476 & $\mathrm{~g} / \mathrm{cm}^{3}$ \\
Melt height, $H$ & 11.95 & 15.47 & $\mathrm{~cm}$ \\
$I^{\prime} / I$ & 0.02997 & 0.01943 & \\
\hline \hline
\end{tabular}




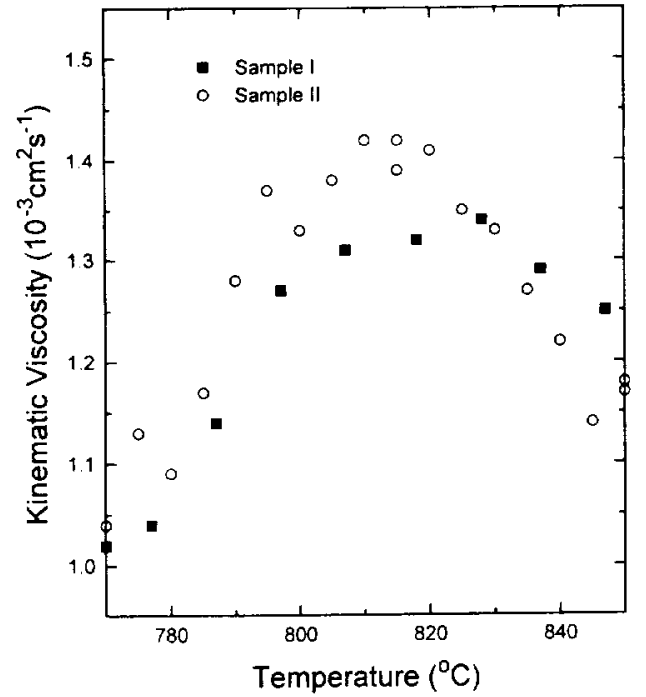

FIG. 3. Kinematic viscosity of the HgZnTe melt for samples I and II.

sample was remeasured after two weeks of soaking inside the rocking furnance at $820^{\circ} \mathrm{C}$, this treatment did not change the observed behavior. A total of 150 measurements were performed for this study and the high temperature viscosity data were reproducible within $5 \%$.

\section{RESULTS AND DISCUSSION}

Figure 3 displays temperature dependence of the kinematic viscosity for both samples. The actual temperature timeline of sample II is presented in Table II. The accuracy of the data has been estimated previously as $5 \%$. The slow relaxation phenomenon is displayed in Fig. 4 where two data sets were obtained by cooling down the second ampoule from $850^{\circ} \mathrm{C}$ and stabilizing at temperatures of 790 and $810^{\circ} \mathrm{C}$. While it took one day for equilibration at $810^{\circ} \mathrm{C}$,

TABLE II. Viscosity data for sample II.

\begin{tabular}{|c|c|c|c|}
\hline $\begin{array}{l}\text { Time } \\
\text { (h) }\end{array}$ & $\begin{array}{c}\text { Temperature } \\
\left({ }^{\circ} \mathrm{C}\right)\end{array}$ & $\begin{array}{c}\text { Logarithmic } \\
\text { decrement }\end{array}$ & $\begin{array}{c}\text { Kinematic } \\
\text { viscosity } \\
\left(10^{-3} \mathrm{~cm}^{2} \mathrm{~s}^{-1}\right)\end{array}$ \\
\hline 2.5 & 850 & 0.01266 & 1.17 \\
\hline 4 & 850 & 0.01269 & 1.18 \\
\hline 6 & 845 & 0.01254 & 1.14 \\
\hline 24 & 840 & 0.01280 & 1.22 \\
\hline 26 & 835 & 0.01298 & 1.27 \\
\hline 30 & 830 & 0.01316 & 1.33 \\
\hline 48 & 825 & 0.01320 & 1.35 \\
\hline 50 & 820 & 0.01338 & 1.41 \\
\hline 53 & 815 & 0.01328 & 1.39 \\
\hline 73 & 815 & 0.01332 & 1.42 \\
\hline 75 & 810 & 0.01341 & 1.42 \\
\hline 78 & 805 & 0.01330 & 1.38 \\
\hline 80 & 800 & 0.01316 & 1.33 \\
\hline 120 & 795 & 0.01326 & 1.37 \\
\hline 123 & 790 & 0.01301 & 1.28 \\
\hline 125 & 785 & 0.01265 & 1.17 \\
\hline 147 & 780 & 0.01237 & 1.09 \\
\hline 149 & 775 & 0.01251 & 1.13 \\
\hline 152 & 770 & 0.01222 & 1.04 \\
\hline
\end{tabular}

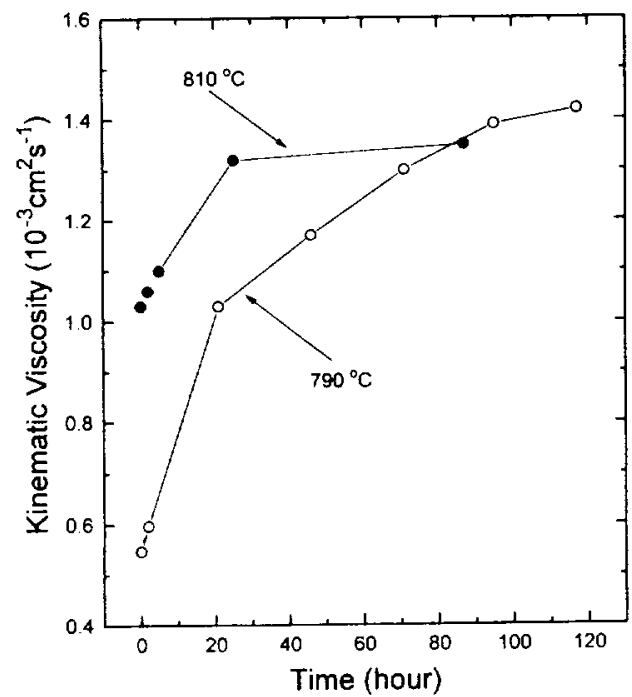

FIG. 4. Time variation of the viscosity after cooling from $850^{\circ} \mathrm{C}$ to the prescribed temperatures, 810 and $790^{\circ} \mathrm{C}$.

more than a week at $790{ }^{\circ} \mathrm{C}$ was required. The average dynamic viscosity of the investigated $\mathrm{HgZnTe}$ melt is approximately $1 \mathrm{cp}$ which falls in the middle of viscosities measured for III-V semiconductor binary melts. ${ }^{6}$ It is about half of that for the $\mathrm{HgCdTe}$ pseudobinary melt and is close to the extrapolated value of a $\mathrm{HgTe}$ melt.

Initial measurements during the course of this work revealed excessive scattering, which was related later to the long time relaxation effect. Possible mechanisms for this observed relaxation of the measured viscosity during temperature cycling might be attributed to either macroscopic or microscopic inhomogeneities.

Macroscopic inhomogeneities can be present, for instance, due to insufficient mixing during material preparation. In fact, any local departure from stoichiometry will significantly change the viscosity, as it has a cusplike sharp peak near the stoichiometric composition. ${ }^{6}$ An inhomogeneity can also arise from evaporation/condensation of mercury into/from a free volume in the ampoule. This could create a thin mercury boundary layer around the sample, thus modifying the wetting condition of the melt to the ampoule walls and changing the surface tension of the top free surface of the melt. It is, however, unlikely that this effect would be present only in the small temperature range of $770-810^{\circ} \mathrm{C}$ and not seen at higher temperatures. Finally, bubble formation in the melt may cause significant effects. Although direct observation of the melt during density measurements ${ }^{10}$ did not show any, the existence of small bubbles is still possible.

The nature of the effect can also be microscopic. Equilibrium density and composition fluctuations near the liquidus point can be qualitatively different from those at higher temperatures, i.e., subcritical clusters of the second phase can be present. ${ }^{7}$ Although the lifetime of these heterofluctuations is finite, it can be quite long. It is conceivable that certain impurities can significantly promote nucleation and alter the equilibrium size and density of these clusters. Also, for complex binary or ternary systems, as compared 
with single-elemental melts, heterofluctuations would more likely be present. If the lifetime of these clusters is significant then any changes in the temperature will induce redistribution in the cluster size and composition. This is a diffusion controlled process which may have similarities to Ostwald ripening phenomenon and can be very slow.

Early viscosity measurements of pure metals showed hysteresis behavior close to their melting points. ${ }^{8}$ Subsequent, and more accurate, measurements did not confirm these findings and the effect was attributed to impurities and surface oxidation. ${ }^{2}$ On the other hand, recent $x$-ray studies of several binary metallic systems revealed a profound long lasting memory effect of the melt microstructure in relation to its thermal history. ${ }^{9}$ However, relevant viscosity studies have not been conducted to date. The viscosity measurements of III-V semiconductor compounds revealed a sharp increase in viscosity near the melting point indicating the presence of some ordering. ${ }^{6}$ Thus, the possibility of slow microscopic relaxation processes taking place in the $\mathrm{HgZnTe}$ melts cannot be ruled out. The temperature dependence of the density of the $\mathrm{HgZnTe}$ melts ${ }^{10}$ also shows a peculiar hysteresis nature, pointing to inhomogeneity of the melt. The present study, unfortunately, is not sufficient to draw any conclusive prediction and more complex work involving $\mathrm{x}$-ray scattering is needed.

\section{ACKNOWLEDGMENT}

This work was supported by the Microgravity Science and Application Division of the National Aeronautics and Space Administration.

${ }^{1}$ K. Mazuruk, C.-H. Su, S. L. Lehoczky, and F. Rosenberger, J. Appl. Phys. 77, 5098 (1995).

${ }^{2}$ H. R. Thresh, Trans. ASME 55, 790 (1962).

${ }^{3}$ J. Kestin and G. F. Newell, J. Appl. Math. Phys. 8, 433 (1957).

${ }^{4}$ R. Roscoe, Proc. Phys. Soc. London 72, 576 (1963).

${ }^{5}$ C.-H. Su, S. L. Lehoczky, and F. R. Szofran, J. Cryst. Growth 86, 87 (1988).

${ }^{6}$ S. Nakamura and T. Hibiya, Int. J. Thermophys. 13, 1061 (1992).

${ }^{7}$ V. I. Yukalov, Phys. Rep. 208, $395(1991)$.

${ }^{8}$ T. P. Yao and V. Kondic, Nature 166, 483 (1950).

${ }^{9}$ V. V. Bukhalenko, S. I. Slyusarenko, V. E. Laz'ko, L. A. Rostovskaya, and O. I. Slukhovskii, Phys. Metals 10, 1025 (1991).

${ }^{10}$ C.-H. Su, Y.-G. Sha, K. Mazuruk, and S. L. Lehoczky, J. Appl. Phys. (submitted). 